\title{
Use of the serum reactivity against Toxoplasma gondii excreted-secreted antigens in cerebral toxoplasmosis diagnosis in human immunodeficiency virus-infected patients
}

Correspondence

Vera L. Pereira-Chioccola

pchioccola@ial.sp.gov.br

Received 11 October 2007

Accepted 7 March 2008

\author{
Cristina S. Meira, ${ }^{1}$ Thais A. Costa-Silva, ${ }^{1}$ José E. Vidal, ${ }^{2}$ \\ Isabelle M. R. Ferreira, ${ }^{1}$ Roberto M. Hiramoto ${ }^{1}$ and Vera L. Pereira-Chioccola ${ }^{1}$ \\ ${ }^{1}$ Department of Parasitology, Instituto Adolfo Lutz, São Paulo, SP, Brazil \\ ${ }^{2}$ Department of Neurology, Instituto de Infectologia Emílio Ribas, São Paulo, SP, Brazil
}

\section{INTRODUCTION}

Toxoplasma gondii is an intracellular parasite that is able to infect all mammalian cells. During the chronic phase, the parasites persist encysted in brain and muscle, and the host develops life-long protective immunity against reinfection (Dubey, 1996, 1998; Hill et al., 2005). The active secretion of antigens by $T$. gondii may be an essential part of the lowgrade stimulation or boosting of the immune system, as these antigens have been shown to stimulate an antibody as well as a T-cell response (Duquesne et al., 1990; Carruthers, 2002). A group of the most important of these antigens has

Abbreviations: ESA, excreted-secreted antigens; HIV, human immunodeficiency virus. been documented as parasite excreted-secreted antigens (ESA), which represent the majority of the circulating antigens in sera from hosts with acute toxoplasmosis (Cesbron-Delauw \& Capron, 1993; Tilley et al., 1997). When released by tachyzoites they are highly immunogenic (Prigione et al., 2000; Carruthers, 2002) and induce protective immunity, either antibody dependent or cell mediated (Zenner et al., 1999). CD4 ${ }^{+} \mathrm{T}$ cells specific for ESA may be involved in the maintenance of long-term immunity in healthy chronically infected individuals. However, when an immunodeficiency appears, such as AIDS, the low levels of $\mathrm{CD}^{+}{ }^{+} \mathrm{T}$ cells cause parasite proliferation and a symptomatic disease such as cerebral toxoplasmosis develops; this being the most frequent 
reactivation of the latent infection (Montoya \& Liesenfeld, 2004; Hoffmann, 2005).

Cerebral toxoplasmosis remains a prevalent disorder of the central nervous system, particularly among severely immunosuppressed human immunodeficiency virus (HIV)-infected patients (Antinori et al., 2004). This situation is critical in resource-limited settings, where the highly active antiretroviral therapy (HAART) is not available. In Brazil, despite a HAART programme being used, cerebral toxoplasmosis still accounts for high mortality and morbidity (Vidal et al., 2005).

In spite of the fact that a definitive diagnosis of cerebral toxoplasmosis requires demonstration of tachyzoites in brain biopsy or necropsy, in clinical practice treatment is usually initiated upon a presumptive diagnosis (based on clinical and radiological features). Recently, the inclusion of PCR in cerebral toxoplasmosis diagnosis has been shown to be an important tool (Cohen, 1999; Bastien, 2002; Bretagne, 2003; Vidal et al., 2004; Colombo et al., 2005), but it is still unavailable in most of the developing countries. As a significant portion of the world population is infected with $T$. gondii, the serological diagnosis is not so relevant in defining a cerebral toxoplasmosis diagnosis, but some studies suggested that high titres might be indicative of the active disease or a higher risk of developing it (Derouin et al., 1996; Hellerbrand et al., 1996; Colombo et al., 2005).

Currently, there is no information about the utility of ESA in distinguishing the cerebral toxoplasmosis in HIVinfected patients. Thus, we evaluated whether sera from patients with cerebral toxoplasmosis specifically recognize ESA and whether this recognition decreases in asymptomatic individuals. The assays were carried out by ELISA using tissue culture cell supernatants infected with tachyzoites RH strain.

\section{METHODS}

Patients and samples. The capability of ESA proteins in distinguishing symptomatic toxoplasmosis was determined by assaying 293 serum samples separated in three groups. Group I, called 'symptomatic sera', was composed of sera from 100 patients with cerebral toxoplasmosis and AIDS. Group II, 'asymptomatic sera', was composed of sera from 99 individuals with asymptomatic toxoplasmosis. Group III, 'control sera', was composed of sera from 94 healthy individuals with negative serology for toxoplasmosis. All HIVinfected patients were admitted and treated at Emilio Ribas Institute of Infectious Diseases, a tertiary teaching hospital in Sao Paulo, Brazil. The sera were previously analyzed by indirect immunofluorescence test and ELISA in the Laboratory of Toxoplasmosis, Instituto Adolfo Lutz. Each serum sample was dissolved in $90 \%$ (v/v) glycerol buffered with $0.5 \mathrm{M}$ carbonate/bicarbonate, $\mathrm{pH} 9.5$, as additive, and stored at $-20{ }^{\circ} \mathrm{C}$ until use. ELISA and immunoblotting standardizations were determined using a panel of 30 well-known sera (from 10 healthy individuals, 10 sera-positive individuals and 10 cerebral toxoplasmosis patients). The diagnosis of cerebral toxoplasmosis in HIV-infected patients was based on: (1) progressive neurological deficits, (2) contrast-enhancing mass lesion(s) on computed tomography scans, and (3) successful response within 2 weeks to specific treatment (Portegies et al., 2004). In addition, all patients had at least one positive PCR of blood and/or cerebrospinal fluid for T. gondii, as previously described (Vidal et al., 2004; Colombo et al., 2005). Blood samples for serological diagnosis and PCR were collected from AIDS patients before or until the third day of the specific therapy for toxoplasmosis. The institutional review boards of the ethics committees of the Instituto de Infectologia Emilio Ribas and Instituto Adolfo Lutz ethically approved this study.

T. gondii and antigens. T. gondii $\mathrm{RH}$ tachyzoites were grown and maintained in Swiss mice ascites by intraperitoneal inoculation. Every 3 or 4 days after infection, the peritoneal fluids from infected mice were collected in PBS, pooled and centrifuged at $1000 \mathrm{~g}$ for $10 \mathrm{~min}$. The parasite pellets were washed twice, counted, and suspended in PBS $\mathrm{pH}$ 7.2, at different concentrations for infecting tissue cultures and preparing antigens for conventional ELISA. Alternatively, tachyzoites were maintained in Vero cells (ATCC CCL - 81), at $37{ }^{\circ} \mathrm{C}, 5 \% \mathrm{CO}_{2}$, in Eagle's medium (Gibco) containing $292 \mathrm{mg} \mathrm{L}-$ glutamine $1^{-1}, 110 \mathrm{mg}$ sodium pyruvate $1^{-1}, 1 \mathrm{~g}$ glucose $\mathrm{l}^{-1}, 2.2 \mathrm{~g}$ sodium bicarbonate $1^{-1}, 100000 \mathrm{U}$ penicillin $\mathrm{l}^{-1}, 133 \mathrm{mg}$ streptomy$\operatorname{cin} 1^{-1}$ and $10 \%$ fetal bovine serum. The tissue culture parasites were used for immunoblotting and the culture infected cell supernatants were used in ESA recovery.

For conventional ELISA, the crude tachyzoite antigen was obtained by sonication of tachyzoites ( 10 cycles of $1.0 \mathrm{~A} \mathrm{~min}^{-1}$, for $5 \mathrm{~min}$, with 2 min intervals). The crude antigen was dissolved in $0.3 \mathrm{M} \mathrm{NaCl}$ and the protein concentration was determined. For immunoblotting, the crude tachyzoite antigen was prepared from $1 \times 10^{7}$ tissue culture tachyzoites solubilized in $50 \mu \mathrm{l}$ SDS-PAGE sample buffer, containing $2 \%$ SDS, $50 \mathrm{mM}$ Tris/ $\mathrm{HCl}, 5 \%$ 2-mercaptoethanol, $10 \%$ (v/v) glycerol, and $0.01 \%$ bromophenol blue, boiled and run in $10 \%$ polyacrylamide-SDS gels. ESA were used in immunoblotting and ELISA (ESA-ELISA). The proteins were recovered from tachyzoiteinfected culture supernatant. For infection with $1 \times 10^{7}$ tachyzoites $\mathrm{ml}^{-1}$, Vero cells were previously washed three times and the medium was replaced by another aliquot of medium without fetal bovine serum. After 48 h post-infection, culture supernatants were harvested, filtered through a $0.22 \mu \mathrm{m}$-pore-size filter and $10 \mu \mathrm{g}$ proteaseinhibitor cocktail $\mathrm{ml}^{-1}$ added, containing (per $\left.\mathrm{ml}\right)$ : $20 \mu \mathrm{m} \mathrm{4-(2-}$ aminoethyl) benzenesulfonyl fluoride, $10 \mu \mathrm{m}$ EDTA, $1.3 \mu \mathrm{m}$ bestatin, $0.14 \mu \mathrm{m}$ E-64, $10 \mathrm{~nm}$ leupeptin, $3 \mathrm{~nm}$ aprotinin (Sigma). The supernatants, referred to as ESA were concentrated in a Speed Vac (RC 10.09; Jouan) for $4 \mathrm{~h}$ and dialysed against PBS overnight at $4{ }^{\circ} \mathrm{C}$. Traces of serum were further observed in ESA preparations, by SDS $10 \%$ PAGE, despite washing the Vero cells before T. gondii infection. Thus, ESA concentrations were determined by dilution for use in ESA-ELISA and immunoblotting.

ELISA and immunoblotting. The sera were tested by ELISA using two antigens. ESA was used at dilution 1:5-1:40 (for each batch of ESA prepared) (ESA-ELISA). The conventional ELISA was carried out using the crude tachyzoite antigen at a concentration of $1 \mu \mathrm{g} \mathrm{ml}^{-1}$ (this antigen is frequently used in conventional diagnosis). The reactions were performed with microtitre polystyrene plates (flat bottom, low binding; Corning). Each plate was incubated overnight at $4{ }^{\circ} \mathrm{C}$ with the wells containing the antigen dissolved in $0.1 \mathrm{ml} 0.1 \mathrm{M} \mathrm{NaHCO}_{3}$, $\mathrm{pH}$ 8.5. Unbound antigen was removed by washing the plates with PBS pH 7.2 containing $0.05 \%$ Tween 20 (PBST). The free binding sites were blocked by treating the wells with $5 \%$ skimmed milk/PBS. After $30 \mathrm{~min}, 50 \mu \mathrm{l}$ each serum sample diluted $1: 200$ in $5 \%$ skimmed milk/ PBS was added to the wells and incubated for $60 \mathrm{~min}$ at $37^{\circ} \mathrm{C}$. After five washes with PBST, the plates were incubated for a further $60 \mathrm{~min}$ at $37{ }^{\circ} \mathrm{C}$ with horseradish peroxidase-conjugated goat anti-human IgG (Sigma) diluted $1: 10000$ in 5\% skimmed milk/PBS. After a new wash cycle with PBST, substrate solution (0.1 M citric acid, $0.2 \mathrm{M} \mathrm{Na}_{2} \mathrm{HPO}_{4}$, $0.05 \% o$-phenylenediamine, $0.1 \% \mathrm{H}_{2} \mathrm{O}_{2}$ ) was added to each well, and the plates were left to stand at room temperature in the dark for 
30 min. Colour development was stopped by adding $50 \mu \mathrm{l} 2 \mathrm{M} \mathrm{H}_{2} \mathrm{SO}_{4}$, and the absorbance was measured with an ELISA reader (Multiscan; Labsystems) with a $492 \mathrm{~nm}$ filter. The reactions were previously standardized with 30 sera described above. Different dilutions of ESA ( $1: 10$ to $1: 80)$ on the microtitre plates, different conjugate dilutions $(1: 2500$ to $1: 20000)$ and different serum dilutions $(1: 50$ to $1: 400)$ were tested (data not shown). Each serum sample was assayed in duplicate. The absorbance values were subtracted from the background, and the arithmetic mean was calculated. The cut-off was calculated in each reaction using a sera panel from 20 healthy individuals.

For immunoblotting, ESA and lysed tachyzoites ( $20 \mu$ l per strip) were boiled and fractionated by run $10 \%$ SDS-PAGE (Laemmli, 1970). The proteins were transferred to nitrocellulose membranes (Towbin et al., 1979), cut into 3-4 mm wide strips, blocked for $1 \mathrm{~h}$ with $5 \%$ skimmed milk PBS and incubated with serum diluted 1:50 at room temperature. After $1 \mathrm{~h}$, the strips were washed with PBS and incubated for $1 \mathrm{~h}$ at room temperature with goat horseradish peroxidase-conjugated anti-human IgG diluted $(1: 500)$ in $5 \%$ skimmed milk/PBS. Bound antibodies were visualized after incubation with $0.03 \%$ 4-chloro-1-naphthol (Sigma) in methanol, $50 \mathrm{mM}$ Tris/ $\mathrm{HCl} \mathrm{pH}$ 7.6, $0.1 \% \mathrm{H}_{2} \mathrm{O}_{2}$.

Data analysis. The cut-off of each reaction corresponded to the mean plus two times the SD of the $A_{492}$. Next, the absorbance results were transformed into ELISA relative values as described previously (Pereira-Chioccola et al., 2003) and shown in Figs 1 and 2. The ELISA relative values represent the ratio of the absorbance of each serum sample at $492 \mathrm{~nm}\left(A_{492}\right)$ to the cut-off value (serum absorbance/ cutoff absorbance). Values greater than 1.0 were considered reactive.

The results were statistically evaluated by the computer program Sigma Plot 9.0. Differences between the means of the ELISA relative values in symptomatic and asymptomatic groups for ESA and crude tachyzoite antigen were tested for significance by using variance analyses, calculated by $F$ test and single factor ANOVA. The coefficient of similarity between the sera groups was calculated by Student's $t$-test (one-tailed and variances). Differences or similarities were considered statistically significant when the $P$ value was $<0.05$.

\section{RESULTS}

The first step was to set up the conditions for ELISA and immunoblotting. The checkerboard assay performed with both antigens in an ELISA determined the optimal working dilutions of the crude tachyzoite antigen $\left(1 \mu \mathrm{g} \mathrm{ml}^{-1}\right)$ and ESA (dilution 1:5-1:40 depending on the batch) in alkaline buffer $\left(0.1 \mathrm{M} \mathrm{NaHCO}_{3}, \mathrm{pH} 8.5\right)$, using the microtitre polystyrene plates type (low binding). Next, the optimal working dilutions were determined for serum samples $(1: 200)$ and conjugate $(1: 10000)$, and the intraand inter-assay reproducibility was determined. These experimental conditions yielded the greatest difference in absorbance between positive and negative samples. For immunoblotting, the standardization assays were performed with ESA. The optimal working dilution for solubilization $(20-30 \mu \mathrm{l})$ was 4:1 in SDS-PAGE sample buffer. The optimal working dilution was also determined for serum samples $(1: 50)$ and conjugate $(1: 500)$.

Next, we analysed the reactivity of the sera for both antigens. The same batch of sera was assayed in parallel for both antigens in order to maintain the same laboratory conditions. The crude tachyzoite antigen is largely used for 'in house' conventional ELISA and commercial kits. We assayed the serum samples from all groups and the results, shown in Fig. 1(a), were expressed in ELISA relative values as described in Methods. The mean ELISA relative value for sera from the control group was $0.5 \pm 0.02$ (mean $\pm \mathrm{SE}$ ). In contrast, the values for serum samples from patients of groups I and II were $8.7 \pm 0.29$ and $8.2 \pm 0.26$, respectively. Student's $t$-test revealed that both groups are statistically similar $(P>0.05)$. As expected, serum samples collected from groups I or II patients were reactive with crude tachyzoite antigen (Fig. 1b, strip 1) in immunoblotting, giving similar intensities (Fig. 1b, strips I and II).

At the same time, we tested the same sera in ESA-ELISA. The ELISA relative values are shown in Fig. 1(c). The mean value for sera from control group were $0.5 \pm 0.02$. Group I (symptomatic) had high values (12.6 \pm 0.74$)$. In contrast, group II (asymptomatic) showed a mean value of $4.2 \pm 0.21$. Student's $t$-test showed that both groups are statistically different $(P<0.05)$. These results were con-

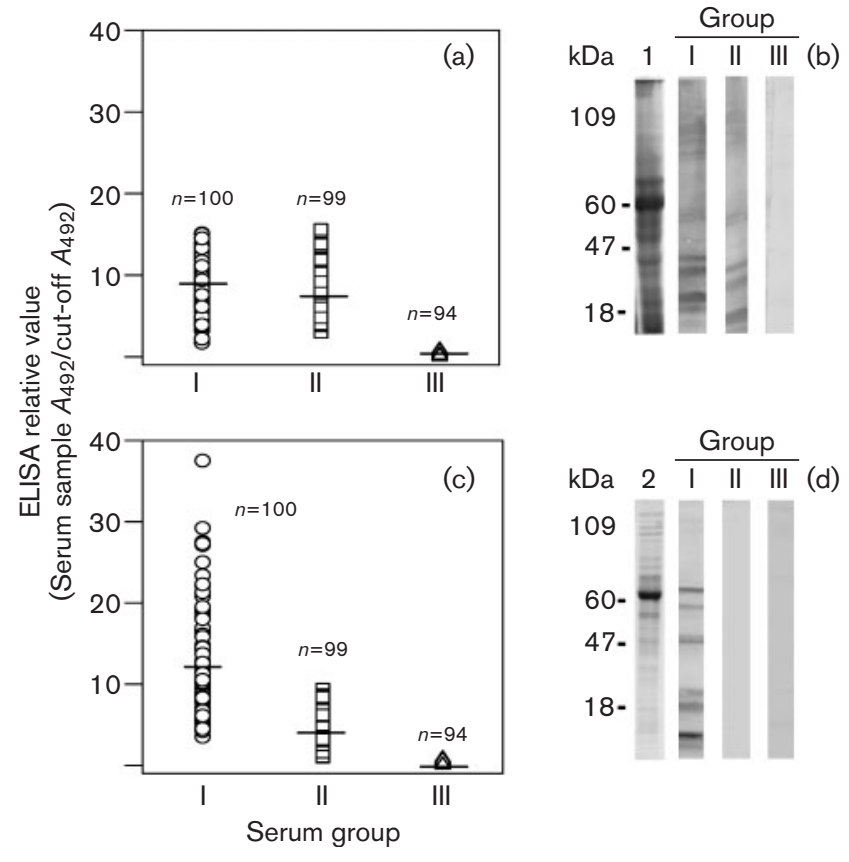

Fig. 1. Serological reactivity of crude tachyzoite lysate (a) and $T$. gondii ESA (c) against sera from patients with cerebral toxoplasmosis and AIDS $(\bigcirc)$, individuals with chronic toxoplasmosis $(\square)$ and healthy individuals $(\triangle)$ by ELISA. The results were calculated as the ratio of the absorbance of each serum sample at $492 \mathrm{~nm}$ to the cut-off absorbance value. Values greater than 1.0 were considered reactive. The horizontal lines represent the arithmetic means: 8.7 and 12.7 in group I, 8.2 and 4.3 in group II, 0.5 and 0.4 in group III. Student's t-test revealed that groups I and II are statistically similar in (a) and different in (c) at $P<0.05$. (b, d) Immunoblotting analysis of a lysate $-1 \times 10^{7}$ tachyzoites (b, strip 1) and ESA (d, strip 2), separated by $10 \%$ SDS-PAGE and transferred to nitrocellulose. Serum from each group (I, II and III) was incubated with the nitrocellulose strips. 
firmed by immunoblotting, as shown in Fig. 1(d). Sera from group I reacted well with ESA (Fig. 1d, strip I), while sera collected from group II failed to do so.

The data presented in Fig. 2 show the distribution of ELISA relative values for sera obtained after testing the reactivity using the crude tachyzoite lysate and ESA in ELISA. The linear regression clearly indicates that the sera from chronic individuals were more reactive against the crude tachyzoite lysate than ESA. The correlation index was 0.48 (Fig. 2a). In contrast, the linear regression shown in Fig. 2(b) indicates that sera from patients with cerebral toxoplasmosis had high reactivity with ESA compared with those with crude tachyzoite lysate. The correlation index was 1.4.

\section{DISCUSSION}

Despite development of serological and molecular methods in recent years, the diagnosis of cerebral toxoplasmosis in

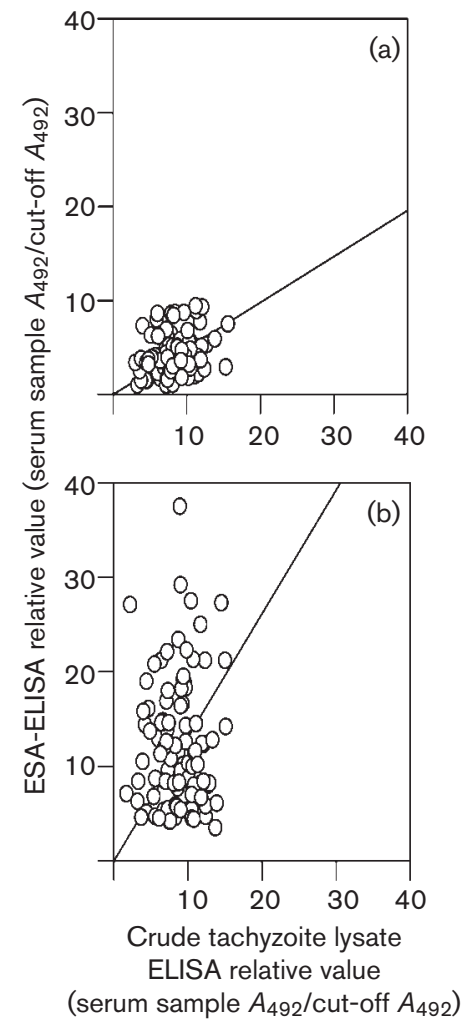

Fig. 2. Correlation between the results of ELISA employing crude tachyzoite lysate and ESA-ELISA. Each point represents the reactivity of each serum sample of chronic individuals (a) and cerebral toxoplasmosis patients (b) using ESA ( $y$-axis) and the crude tachyzoite lysate antigen ( $x$-axis). Each serum result was calculated as the ratio of the absorbance of each serum sample at $492 \mathrm{~nm}\left(A_{492}\right)$ to the cut-off value. Values greater than 1.0 were considered reactive. $r$ (the correlation index for each test) was 0.48 and 1.4 for (a) and (b), respectively.
HIV-infected patients still presents difficulties. In clinical practice, specific treatment is usually initiated in response to a presumptive diagnosis, which is based on clinical and radiological features (Luft \& Remington, 1992). Thus, the employment of the $T$. gondii proteins of the serological diagnosis seems to be a promising tool.

It is known that $T$. gondii invasion initiates a lytic cycle leading to cell and tissue destruction that is a hallmark feature of Toxoplasma pathology. This process is rapid, dynamic, and relies on the secretion of numerous secretory proteins from micronemes, rhoptries and dense granules (Carruthers, 2002). Despite significant progress in studying these proteins, only a limited number of secretory proteins have been discovered (ToxoDB: the $T$. gondii genome resource, accessed in September 2007; http://www.toxodb. org/toxo/home.jsp) (Zhou et al., 2005). In addition, as we showed recently, a great genetic variability has been observed between the $T$. gondii isolates of patients (Ferreira et al., 2008). For this reason, we decided to use pooled ESA recovered from tachyzoite-infected culture supernatants as antigen because each protein could elicit the host immune system by antibody production.

The vast majority of infected immunocompetent individuals with latent infection by toxoplasmosis remain asymptomatic. Despite the presence of T. gondii antibodies, they have no or a low number of circulating tachyzoites. In general, these antibodies are determined in conventional serology, using total extract of tachyzoites as antigen, which consists of cytoplasmic and membrane components. When we used this antigen (crude tachyzoite antigen) in ELISA, similar reactivity was shown between sera from patients with cerebral toxoplasmosis and those from patients with latent infection (mean value of the ratio of the relative absorbance). These results suggest that this antigen, widely used in conventional diagnosis, failed to distinguish the two groups of sera.

In an earlier study we have shown that the majority of the patients with cerebral toxoplasmosis have high anti- $T$. gondii $\operatorname{IgG}$ titres with high avidity, suggesting that the reactivation of the latent infection observed in immunocompromised patients occurs in the secondary immune response (Colombo et al., 2005). In this phase, numerous tachyzoites are released from the quiescent cysts and a considerable proportion of ESA are released, eliciting the specific immune response to these antigens. In this case, these patients present antibodies for both ESA and total crude tachyzoites antigens. ESA-ELISA distinguished sera from patients with the active disease (cerebral toxoplasmosis and positive PCR). These sera were three times more reactive than those from seropositive individuals (12.6 to 4.2). These data were reproducible based on the immunoblotting results, showing that ESA constitute an excellent serological marker for the diagnosis of cerebral toxoplasmosis in HIV-infected patients.

Even though the majority of patients with cerebral toxoplasmosis develop high titres of anti-T. gondii 
antibodies (Derouin et al., 1996; Hellerbrand et al., 1996; Colombo et al., 2005), some also show a humoral response deficiency and no antibodies are detected (Luft \& Remington, 1992; Skiest, 2002; Vidal et al., 2005). In this study, six patients with cerebral toxoplasmosis confirmed by a positive PCR of blood had a negative immunofluorescence test (for both IgG and IgM antibodies) and ELISA (both antigens) (data not shown). In this case ESA-ELISA also failed in diagnosing the active infection, confirming the caveats of serological diagnosis in a reduced subset of patients.

In summary, our results suggest that anti-ESA antibodies are present in patients with HIV-related cerebral toxoplasmosis, whereas the absence of a significant amount of antibodies distinguished the patients without clinical symptoms of infection. ESA-ELISA can be used for the diagnosis of cerebral toxoplasmosis in association with clinical and radiological information, providing a simple methodology to be used in countries with high prevalence of latent toxoplasmosis in the general population.

\section{ACKNOWLEDGEMENTS}

This study was supported by grants from the FAPESP (Fundaçao de Amparo à Pesquisa do Estado de Sao Paulo, Brazil), proc-05/03052-5. I. M. R. F., C. S. M. and T.A.C. S. were supported by fellowships from FAPESP: proc-06/52672-9, 06/52674-1 and 06/52673-5.

\section{REFERENCES}

Antinori, A., Larussa, D., Cingolani, A., Lorenzini, P., Bossolasco, S., Finazzi, M.G., Bongiovanni, M., Guaraldi, G., Grisetti, S. \& other authors (2004). Prevalence, associated factors, and prognostic determinants of AIDS-related toxoplasmic encephalitis in the era of advanced highly active antiretroviral therapy. Clin Infect Dis 39, 1681-1691.

Bastien, P. (2002). Molecular diagnosis of toxoplasmosis. Trans R Soc Trop Med Hyg 96 (Suppl. 1), S205-S215.

Bretagne, S. (2003). Molecular diagnostics in clinical parasitology and mycology: limits of the current polymerase chain reaction (PCR) assays and interest of the real-time PCR assays. Clin Microbiol Infect 9, 505-511.

Carruthers, V. B. (2002). Host cell invasion by the opportunistic pathogen Toxoplasma gondii. Acta Trop 81, 111-122.

Cesbron-Delauw, M. F. \& Capron, A. (1993). Excreted-secreted antigens of Toxoplasma gondii: their origin and role in the hostparasite interaction. Res Immunol 144, 41-44.

Cohen, B. A. (1999). Neurological manifestations of toxoplasmosis in AIDS. Semin Neurol 19, 201-211.

Colombo, F. A., Vidal, J. E., Penalva de Oliveira, A. C., Hernández, A. V., Bonasser-Filho, F., Nogueira, R. S., Focaccia, R. \& PereiraChioccola, V. L. (2005). Diagnosis of cerebral toxoplasmosis in AIDS patients in Brazil: importance of molecular and immunological methods using peripheral blood samples. J Clin Microbiol 43, 5044 5047.

Derouin, F., Leport, C., Pueyo, S., Morlat, P., Letrillart, B., Chene, G., Ecobichon, J. L., Luft, B., Aubertin, J. \& other authors (1996). Predictive value of Toxoplasma gondii antibody titres on the occurrence of toxoplasmic encephalitis in HIV-infected patients. AIDS 10, 1521-1527.
Dubey, J. P. (1996). Toxoplasma gondii. In Medical Microbiology, 5th edn. Edited by S. Baron, R. C. Peake, D. A. James, M. Susman, C. A. Kennedy, M. J. D. Singleton \& S. Schuenke. Galveston, TX: University of Texas Medical Branch.

Dubey, J. P. (1998). Advances in the life cycle of Toxoplasma gondii. Int J Parasitol 28, 1019-1024.

Duquesne, V., Auriault, C., Darcy, F., Decavel, J. P. \& Capron, A. (1990). Protection of nude rats against Toxoplasma infection by excreted-secreted antigen-specific helper T-cells. Infect Immun 58, 2120-2126.

Ferreira, I. M. R., Vidal, J. E., Costa-Silva, T. A., Meira, C. S., Hiramoto, R. M., Penalva de Oliveira, A. C. \& Pereira-Chioccola, V. L. (2008). Toxoplasma gondii: genotyping of strains from Brazilian AIDS patients with cerebral toxoplasmosis by multilocus PCR-RFLP markers. Exp Parasitol 118, 221-227.

Hellerbrand, C., Goebel, F. D. \& Disko, R. (1996). High predictive value of Toxoplasma gondii IgG antibody levels in HIV-infected patients for diagnosis of cerebral toxoplasmosis. Eur J Clin Microbiol Infect Dis 15, 869-872.

Hill, D. E., Chirukandoth, S. \& Dubey, J. P. (2005). Biology and epidemiology of Toxoplasma gondii in man and animals. Anim Health Res Rev 6, 41-61.

Hoffmann, C. (2005). Opportunistic infections. In HIV Medicine pp. 365-344. Edited by C. Hoffmann, J. K. Rockstroh \& B. S. Kamps. Paris: Flying Publisher. www.HIVMedicine.com.

Laemmli, U. K. (1970). Cleavage of structural proteins during the assembly of the head of bacteriophage T4. Nature 227, 680-681.

Luft, B. J. \& Remington, J. S. (1992). Toxoplasmic encephalitis. Clin Infect Dis 15, 211-222.

Montoya, J. G. \& Liesenfeld, O. (2004). Toxoplasmosis. Lancet 363, 1965-1976.

Pereira-Chioccola, V. L., Fragata-Filho, A. A., Levy, A. M., Rodrigues, M. M. \& Schenkman, S. (2003). Enzyme-linked immunoassay using recombinant trans-sialidase of Trypanosoma cruzi can be employed for monitoring of patients with Chagas' disease after drug treatment. Clin Diagn Lab Immunol 10, 826-830.

Portegies, P., Solod, L., Cinque, P., Chaudhuri, A., Begovac, J., Everall, I., Weber, T., Bojar, M., Martinez-Martin, P. \& Kennedy, G. E. (2004). Guidelines for the diagnosis and management of neurological complications of HIV infection. Eur J Neurol 11, 297-304.

Prigione, I., Facchetti, P., Lecordier, L., Deslee, D., Chiesa, S., Cesbron-Delauw, M. F. \& Pistoia, V. (2000). T cell clones raised from chronically infected healthy humans by stimulation with Toxoplasma gondii excretory-secretory antigens cross-react with live tachyzoites: characterization of the fine antigenic specificity of the clones and implications for vaccine development. J Immunol 164, 3741-3748.

Skiest, D. J. (2002). Focal neurological disease in patients with acquired immunodeficiency syndrome. Clin Infect Dis 34, 103-115.

Tilley, M., Fichera, M. E., Jerome, M. E., Roos, D. S. \& White, M. W. (1997). Toxoplasma gondii sporozoites from a transient parasitophorus vacuole that is impermeable and contains only a subset of dense granule proteins. Infect Immun 65, 4598-4605.

Towbin, H., Staehelin, T. \& Gordon, J. (1979). Electrophoretic transfer of proteins from polyacrylamide gels to nitrocellulose sheets: procedure and some applications. Proc Natl Acad Sci U S A 76, 4350-4357.

Vidal, J. E., Colombo, F. A., Penalva de Oliveira, A. C., Focaccia, R. \& Pereira-Chioccola, V. L. (2004). PCR assay using cerebrospinal fluid for diagnosis of cerebral toxoplasmosis in Brazilian AIDS patients. J Clin Microbiol 42, 4765-4768. 
Vidal, J. E., Hernandez, A. V., Penalva de Oliveira, A. C., Dauar, R. F., Barboza, S. P. Jr \& Focaccia, R. (2005). Cerebral toxoplasmosis in HIVpositive patients in Brazil: clinical features and predictors of treatment response in the HAART era. AIDS Patient Care STDS 19, 626-634.

Zenner, L., Estaquier, J., Darcy, F., Maes, P., Capron, A. \& CaesbronDelauw, M. F. (1999). Protective immunity in the rat model of congenital toxoplasmosis and the potential vaccinal role of excretedsecreted antigens (ESA). Parasite Immunol 21, 261-272.

Zhou, X. W., Kafsack, B. F. C., Cole, R. N., Beckett, P., Shen, R. F. \& Carruthers, V. B. (2005). The opportunistic pathogen Toxoplasma gondii deploys a diverse legion of invasion and survival proteins. J Biol Chem 280, 34233-34244. 